\title{
All-cis 1,2,3,4,5,6-hexafluorocyclohexane is a facially polarised cyclohexane
}

\author{
Neil S. Keddie, Alexandra M. Z. Slawin, Tomas Lebl, Douglas Philp, David O’Hagan^ \\ EaStCHEM School of Chemistry, University of St Andrews, North Haugh, St Andrews, KY16 9ST, UK. \\ *e-mail: do1@st-andrews.ac.uk
}

The highest energy stereoisomer of 1,2,3,4,5,6-hexafluorocyclohexane, where all of the fluorines are 'up', is prepared in a 12-step protocol. The molecule adopts a classic chair conformation with alternate C-F bonds aligned triaxial, clustering three highly electronegative fluorine atoms in close proximity. This generates a cyclohexane with a high molecular dipole ( $\mu=6.2 \mathrm{D}$ ) unusual in an otherwise aliphatic compound. X-ray analysis indicates that the intramolecular $F_{a x} \ldots F_{a x}$ distances $(-2.77 \AA)$ are longer than the vicinal $F_{a x} \ldots F_{\text {eq }}$ distances $(-2.73 \AA)$ suggesting a tension stabilising the chair conformation. In the solid state the molecules pack in an orientation consistent with electrostatic ordering. Our synthesis of this highest energy isomer demonstrates the properties that accompany the placement of axial fluorines on a cyclohexane and the unusual property of a facially polarised ring in organic chemistry. Derivatives have potential as new motifs for the design of functional organic molecules or for applications in supramolecular chemistry design.

Fluorine is frequently used to modulate the properties of organic bioactives in pharmaceuticals and agrochemicals ${ }^{1,2}$ and it also confers attractive properties of chemical resistance to polymers (eg. poly-tetrafluoroethylene) or polarity to piezoelectric materials such as poly-vinylidene fluoride and organic liquid crystals for displays ${ }^{3}$. The $\mathrm{C}-\mathrm{F}$ bond is the most polarised, and strongest in organic chemistry due to the highest electronegativity of fluorine ${ }^{4}$. This renders fluorine a weaker hydrogen bonding acceptor and thus organofluorines are poorly solvated in water, possessing 'polar hydrophobic' properties ${ }^{5}$. In linear alkanes carrying fluoromethylene carbons $(\mathrm{CHF}), \mathrm{C}-\mathrm{F}$ bonds positioned on alternate carbons tend to orientate away from each other to minimise dipolar repulsion, an aspect that compromises the overall molecular dipole moment ${ }^{6,7}$. However, if such 1,3-alignments could be maintained, polar organic molecules would result. The cyclohexane ring is a core structural motif in organic chemistry defined by its clear conformational preference and 
hydrophobic nature ${ }^{8}$. In this paper we report the synthesis and structure of all-cis $1,2,3,4,5,6-$ hexafluorocyclohexane 1, a cyclohexane that contains a fluorine atom on each carbon and with a stereochemistry that has all of the fluorines on one face of the cyclohexane ring.

Progress towards this goal has been demonstrated for two of the isomers of all-cis tetrafluorocyclohexane, where two diaxial $\mathrm{C}-\mathrm{F}$ bonds align and that gave rise to crystalline materials with dipole moments of $\mu \sim 4.9-5.2 \mathrm{D}^{9,10}$. Our goal was to extend to a $1,2,3,4,5,6-$ hexafluorocyclohexane. There are 9 possible configurational isomers with 15 conformations in total of 1,2,3,4,5,6-hexafluorocyclohexane, if the rings adopt a chair conformation (Fig. 1). The '4 up 2 down' structure (v) was recently prepared and characterised in our $1 \mathrm{lab}^{11}$. Previous theoretical analyses performed at the DF-MP2/cc-pVTZ ${ }^{12}$ and MP2/6-31++G ${ }^{13}$ levels of theory, evaluated relative energies of all of the isomers and in each case revealed that the ' 6 up' isomer 1 (viii) is a stand out structure almost twice the energy of the next nearest isomer in the series, due to the unique aspect of the 1,3,5-triaxial $\mathrm{C}-\mathrm{F}$ bonds, rendering it particularly polar.

At the outset it was not clear whether isomer $\mathbf{1}$ could be prepared synthetically. The strategy envisaged the stereospecific conversion of $\mathrm{C}-\mathrm{O}$ to $\mathrm{C}-\mathrm{F}$ bonds via $\mathrm{S}_{\mathrm{N}} 2$ reactions occurring with inversions of configuration. However, due to the steric and electronic repulsion required to overcome placing three axial $\mathrm{C}-\mathrm{F}$ bonds on a cyclohexane framework, it seemed entirely plausible that elimination rather than substitution may follow lower energy pathways in the last step of the synthesis (Fig. 2).

\section{Results and Discussion}

Synthesis. The route required a precursor with six vicinal C-Os all with the 'down' stereochemistry. cis-Dianhydroinositol 3 was prepared as a candidate precursor from myoinositol 2 by modification of previously described approaches (Supplementary Figure 1) $)^{14}$. The stereochemistry of $\mathbf{3}$ was confirmed by X-ray structure analysis (Supplementary page 
29), which showed all of the oxygen atoms configured cis around the ring. The high symmetry of $\mathbf{3}$ allowed the fluorines to be introduced in pairs over the next two steps. Treatment of 3 with Deoxofluor ${ }^{\mathrm{TM}} 15$ afforded $\mathbf{4}$ with both epoxide rings intact, using either conventional $\left(90{ }^{\circ} \mathrm{C}, 18 \mathrm{~h}\right)$ or microwave-assisted $\left(60-100{ }^{\circ} \mathrm{C}\right.$ over $\left.15 \mathrm{~min}\right)$ heating. The epoxides were then opened with $3 \mathrm{HF} \cdot \mathrm{Et}_{3} \mathrm{~N}\left(180{ }^{\circ} \mathrm{C}, 2 \mathrm{~h}\right)$, also in a microwave-assisted reaction, to afford tetrafluorodiol $\mathbf{5}$ as a single isomer as confirmed by X-ray structure analysis (Supplementary Information page 30). The four fluorine atoms are all-cis to each other, and trans to the remaining hydroxyl groups. No trace of alternative regioisomers was observed. The final two deoxofluorination reactions were now explored and in the end were completed sequentially rather than in a single transformation. Deoxofluorination of $\mathbf{5}$ under a range of conditions and temperatures with $\mathrm{DAST}^{16}$, Deoxofluor ${ }^{\mathrm{TM}}$ and XtalFluor-E ${ }^{\circledR 17}$ all failed to progress the material to higher fluorinated products. The use of Deoxofluor ${ }^{\mathrm{TM}}$ at higher temperature $\left(\sim 120^{\circ} \mathrm{C}\right)$ produced only traces of pentafluoroalcohol 7 , however, with an insufficient conversion to be viable. Progress required monotriflation ( $89 \%$ yield) to generate 6 and then displacement by fluoride ion using $3 \mathrm{HF} \cdot \mathrm{Et}_{3} \mathrm{~N}$ at $120^{\circ} \mathrm{C}$. Again microwave assisted heating proved more practical than conventional heating and gave pentafluoroalcohol 7 (39\% yield). Stereochemical inversion was again confirmed by X-ray structure analysis (Supplementary Information page 30). Activation of the remaining alcohol moiety, also as a triflate, generated 8. In order to introduce the final fluorine atom, $\mathbf{8}$ was treated with $3 \mathrm{HF} \cdot \mathrm{Et}_{3} \mathrm{~N}$ under heated microwave conditions. Not unexpectedly, this reaction was very sluggish, but it gave rise to three new products in addition to unreacted $\mathbf{8}$. The reaction was followed by ${ }^{19} \mathrm{~F}\left\{{ }^{1} \mathrm{H}\right\}$ NMR (Fig 3.).

The major component of the mixture was pentafluoroalcohol 7 , which presumably arises from fluorolysis, rather than fluoride ion displacement of the triflyl group. This is an unusual reaction course for a triflate, one of the best leaving groups in organic chemistry and 
indicates the difficulty in achieving the final substitution reaction. Also a pentafluoro alkene (structure not unambiguously determined) is present, which presumably arose from fluoride ion induced elimination of the triflate. However, analysis of the product mixture by ${ }^{19} \mathrm{~F}$ NMR indicated that the desired cis-hexafluorocyclohexane $\mathbf{1}$ was present as a minor component and it was isolated after careful chromatography.

Structure analysis. X-ray structure analysis of a suitable crystal of $\mathbf{1}$ reveals a classic chair conformation for the cyclohexane and as a consequence six alternating axial and equatorial C-F bonds (Fig. 4). A calculated (M11/6-311G(2d,p)) electrostatic potential surface profile $^{18,19}$ illustrates very clearly that $\mathbf{1}$ is facially polarised (Fig. 4c). The axial fluorines present a negative face and the axial hydrogens a positive face, a bipolar aspect unusual in an organic motif. From the X-ray structure, the intramolecular axial/equatorial vicinal $\mathrm{F}$...F distances $(\sim 2.73 \AA)$ are actually shorter than the triaxial F...F distances $(\sim 2.77 \AA)$ suggesting a slightly greater tension between the axial/equatorial fluorines rather than axial/axial fluorines. This also manifests in the $\mathrm{C}-\mathrm{C}-\mathrm{C}$ angles, which alternate wider $\left(\sim 114^{\circ}\right) /$ narrower $\left(\sim 109^{\circ}\right)$ around the ring, the wider angles having an equatorial fluorine on the central carbon, suggesting that these fluorines are forced back with the three axial fluorines becoming aligned, increasing the overall molecular dipole. This tensioning supports a chair over a more splayed open structure. The molecular packing observed in the X-ray structure of 1 reveals that the individual molecules stack on top of each other in a manner consistent with electrostatic attraction between the non-equivalent faces of the cyclohexanes, rather than perhaps an alternative 'back to back' mode between like 'fluorous' and 'hydrocarbon' faces $(\text { Fig. } 5)^{20}$.

The structure of $\mathbf{1}$ has some analogy to the recently described ${ }^{21}$ two dimensional (2D) $C_{2} F$ chair representation of partially fluorinated graphene (Fig. 6) as determined by electron microscopy. In that representation there are ordered domains of 1,3,5-triaxial fluorines on a 
chair cyclohexane. The $\mathrm{C}_{2} \mathrm{~F}$ chair represented in Figure 6 is predicted to be favoured over other candidates ${ }^{22}$ and has axial fluorines only on one face and more compacted $\left(\mathrm{d}_{\mathrm{FF}}=2.52\right.$ $\AA)$ than that found in $\mathbf{1}\left(\mathrm{d}_{\mathrm{FF}} \sim 2.77 \AA\right)$, certainly in the idealised lattice structure. The nonfluorinated carbons of the $\mathrm{C}_{2} \mathrm{~F}$ structure have incomplete valencies and the material is predicted to be metallic. Also it is predicted that if hydrogen atoms adsorb to the lower face it will become positively polarised and the material will possess a permanent transverse electric field ${ }^{32}$. Our observations for $\mathbf{1}$ are clearly consistent with such a prediction.

EXSY ${ }^{19}$ F NMR experiments. Variable temperature NMR studies were conducted to measure the energy barrier to ring-flipping for $\mathbf{1}$ in solution. Thermodynamic parameters for the degenerate ring-flip were calculated using ${ }^{19} \mathrm{~F}$ EXSY (exchange correlation spectroscopy) $(470.6 \mathrm{MHz})(\text { Supplementary Figure 3) })^{23}$. Data points were measured between $241 \mathrm{~K}$ and 273 $\mathrm{K}$, from which an Eyring plot was constructed (Supplementary Figure 4). Extrapolation of the plot, calculated the barrier to degenerate ring-flip to be $\Delta \mathrm{H}^{*}=13.3 \pm 0.43 \mathrm{kcal} \mathrm{mol}^{-1}, \Delta \mathrm{S}^{\ddagger}=$ $3.8 \pm 1.6 \mathrm{cal} \mathrm{mol}^{-1} \mathrm{~K}^{-1}$, which is only a little higher than cyclohexane itself $\left(\Delta \mathrm{H}^{\ddagger}=10.8 \mathrm{kcal}\right.$ $\left.\mathrm{mol}^{-1}, \Delta \mathrm{S}^{\ddagger}=2.8 \mathrm{cal} \mathrm{mol}^{-1} \mathrm{~K}^{-1}\right)^{24}$, and suggests that the ground state chair and transition structure of $\mathbf{1}$ are both significantly raised relative to cyclohexane.

Theoretical calculations. A molecular dipole value of 6.2 D for 1 was calculated at M11/6$311 \mathrm{G}(2 \mathrm{~d}, \mathrm{p})$ theory level using NBO $6.0^{25-28}$. This value was calibrated against the calculated values for dimethyl sulfoxide $(4.07 \mathrm{D})$ and fluoroform $(1.68 \mathrm{D})$ at the same theory level, which are in good agreement with their experimentally measured values (3.96 D and $1.65 \mathrm{D}$ respectively $)^{29,30}$. The magnitude of the molecular dipole of $\mathbf{1}$ is the highest we can identify for a non-ionic, aliphatic or aromatic organic compound. E.g. it is more polar than 2pyridinecarbonitrile $(5.78 \mathrm{D}), 2$-methoxynitrobenzene $(5.0 \mathrm{D}), \mathrm{N}$-methylacetamide $(4.3 \mathrm{D})$, and benzonitrile $(4.18 \mathrm{D})^{29,30}$. In terms of aliphatics, the previously prepared all-cis $1,2,3,4-$ and 1,2,4,5- tetrafluorocyclohexanes (calculated at $4.91 \mathrm{D}$ and 5.24 $\mathrm{D}$ respectively) ${ }^{9,10}$, and 
ethylene carbonate (4.9 D measured $)^{29}$ have the closest molecular dipole values to $\mathbf{1}$. Thus $\mathbf{1}$ appears to be a privileged compound in terms of its high molecular dipole moment. Related characteristics are found in poly-vinylidine fluoride (PVDF) the well-described piezoelectric polymer which displays a high dipole moment when the $\mathrm{CF}_{2}$ groups are aligned (poled) in an electric field ${ }^{31}$. The orientation of the $\mathrm{CF}_{2}$ groups antiparallel to the $\mathrm{CH}_{2}$ groups in the extended polymer chain maximises the molecular dipole of PVDF.

Finally, natural bond orbital (NBO) analysis using NBO 6.0 $25-28$ (M11/6-311G(2d,p) theory level) (Table 1, Supplementary Table 1 and Supplementary Figure 5) provides some insight into the bonding in $\mathbf{1}$, and, in particular, the principal interactions between fluorine lone pairs and other orbitals. For the axial fluorines, primary hyperconjugative interactions exist between two of the lone pairs and antibonding $\left(\sigma^{*}\right)$ orbitals associated with the geminal $\mathrm{C}-\mathrm{C}$ and $\mathrm{C}-\mathrm{H}$ bonds. The lone pair $\left(\mathrm{LP}^{3}\right)$ that interacts with the $\mathrm{C}-\mathrm{C}$ bonds distributes almost equally into both geminal $\sigma^{*} \mathrm{C}-\mathrm{C}$ orbitals. Similar interactions, although slightly weaker, exist between the lone pairs associated with the equatorial $\mathrm{F}$ atoms and the corresponding $\sigma^{*}(\mathrm{C}-\mathrm{C})$ and $\sigma^{*}(\mathrm{C}-\mathrm{H})$ orbitals. Significant interactions also exist between the $\sigma$ orbitals associated with the axial $\mathrm{C}-\mathrm{H}$ bonds and the $\sigma^{*}$ orbitals of the adjacent antiperiplanar $\mathrm{C}-\mathrm{F}$ bonds. Although these interactions account for the classical gauche effect ${ }^{32}$ in 1,2difluoroethane, in this case they are somewhat less significant than those involving fluorine lone pair dispersion. 


\section{Conclusion}

The synthesis of the highest energy, all-cis isomer of 1,2,3,4,5,6-hexafluorocyclohexane 1 demonstrates that it is possible to assemble a molecule with three triaxial $\mathrm{C}-\mathrm{F}$ bonds as a design feature in a non perfluorous material. Benzene and hexafluorobenzene are known to pack in alternating stacks in the solid state due to the complementary electrostatics of each ring $^{33,34}$, however, the polarised faces of hexafluorocyclohexane 1 presents a fusion of that arrangement where a single ring has differentially polarised faces. Derivatives of such a fluorinated ring system present a novel design and should impart particular properties when incorporated into higher molecular architectures for applications ranging from organic materials to bioactives or as a single ring with complementary faces for exploration in supramolecular chemistry.

\section{Methods}

Microwave-assisted fluorination of 3 with Deoxofluor. Dianhydroinositol 3 (390 mg, $2.7 \mathrm{mmol}, 1 \mathrm{eq})$ was dissolved in a solution of Deoxofluor in THF $(50 \% \mathrm{w} / \mathrm{v}, 2.58 \mathrm{~mL}$, $6.0 \mathrm{mmol}, 2.2 \mathrm{eq})$ and was heated by microwave irradiation $(50 \mathrm{~W}$, temperature ramp 60 $100^{\circ} \mathrm{C}$ ) in a Teflon flask for $20 \mathrm{~min}$. The reaction mixture was cooled to RT and the resulting dark solution was purified directly by silica gel chromatography, eluting with dichloromethane, to afford 4 (376 mg, 94\%) as a colourless oil.

Microwave-assisted fluorination with $\mathbf{3 H F} \cdot \mathbf{E t}_{3} \mathbf{N} .8(14.0 \mathrm{mg}, 0.04 \mathrm{mmol})$ was dissolved in triethylamine trihydrofluoride $(1 \mathrm{~mL})$ and heated by microwave irradiation $\left(50 \mathrm{~W}, 120{ }^{\circ} \mathrm{C}\right)$ in a Teflon flask for $2 \times 2 \mathrm{~h}$. The resulting sticky brown material was reconstituted with ethyl acetate $(10 \mathrm{~mL})$ and poured into saturated aqueous sodium hydrogen carbonate solution $(20 \mathrm{~mL})$. The layers were separated, and the aqueous phase was re-extracted with ethyl acetate $(3 \times 10 \mathrm{~mL})$. The combined organic phase was washed with brine $(10 \mathrm{~mL})$, dried over 
$\mathrm{MgSO}_{4}$, filtered and concentrated in vacuo, affording a brown gum. Purification of the gum by repeated silica gel chromatography, eluting with dichloromethane and methanol (98:2), furnished 1 ( $c a .0 .8 \mathrm{mg}, 10 \%$ ) as a gum. Crystals suitable for single crystal X-ray structure determination were obtained by slow evaporation of a solution of 1 in $\mathrm{CD}_{2} \mathrm{Cl}_{2}$.

\section{EXSY NMR Spectra Analysis to determine thermodynamic parameters of degenerate}

ring inversion of $1 .{ }^{19} \mathrm{~F}$ NMR EXSY spectra of 1 in $\mathrm{CD}_{2} \mathrm{Cl}_{2}$ were recorded at $470 \mathrm{MHz}$ on a Bruker Avance 500 spectrometer equipped with a variable temperature $5 \mathrm{~mm}$ QNP probe across the temperature range $247-273 \mathrm{~K}$. The accurate temperatures for each data point were determined using a standard sample of $4 \% \mathrm{MeOH}$ in $\mathrm{d}_{4}-\mathrm{MeOD}$. Complete lineshape analysis was carried out using Bruker Topspin D-NMR module. Further details and the thermodynamic parameter calculations are provided in the Supplementary Information, pages $33-34$.

\section{References}

1. Fier, P. S., Hartwig, J. F. Selective C-H fluorination of pyridines and diazines inspired by a classic amination reaction. Science 342, 956-960 (2013).

2. Müller, K., Faeh, C., Diederich, F. Fluorine in pharmaceuticals: looking beyond intuition. Science 317 1881-1886 (2007).

3. Kirsch, P. Modern Fluoroorganic Chemistry: Synthesis, Reactivity, Applications (WileyVCH, Weinheim, ed. 2, 2013).

4. O'Hagan, D. Understanding organofluorine chemistry. An introduction to the $\mathrm{C}-\mathrm{F}$ bond. Chem. Soc. Rev. 37, 308-319 (2008). 
5. Biffinger, J. C., Kim, H. W., DiMagno, S. G. The polar hydrophobicity of fluorinated compounds. ChemBioChem 5, 622-627 (2004).

6. Burns, M. et al. Assembly-line synthesis of organic molecules with tailored shape. Nature 513, 183-188 (2014).

7. Hunter, L., Kirsch, P., Slawin, A. M. Z., O’Hagan, D. Synthesis and structure of stereoisomeric multivicinal hexafluoroalkanes. Angew. Chem. Int. Ed. 48, 5457-5460 (2009).

8. Barton, D. H. R. The conformation of the steroid nucleus. Experientia 6, 316-320 (1950).

9. Durie, A. J., Slawin, A. M. Z., Lebl, T., Kirsch, P., O’Hagan, D. Synthesis and structure of all-syn-1,2,3,4-tetrafluorocyclohexane. Chem Commun. 47, 8265-8267 (2011).

10. Durie, A. J., Slawin, A. M. Z., Lebl, T., Kirsch, P., O’Hagan, D. Fluorocyclohexanes: synthesis and structure of all-syn-1,2,4,5-tetrafluorocyclohexane. Chem Commun. 48, 9643-9645 (2012).

11. Durie, A. J., Slawin, A. M. Z., Lebl, T., O’Hagan, D. The synthesis of $\eta-1,2,3,4,5,6-$ hexafluorocyclohexane (benzene hexafluoride) from benzene. Angew. Chemie. Int. Ed. 51, 10086-10088 (2012).

12. Luo, Q., Randall, K. R., Schaefer, H. F. Easy chairs: the conformational preference of polyfluorinated cyclohexanes. RSC $A d v$. 3, 6572-6585 (2013).

13. Zdravkovski, Z. Theoretical study of the stability of hexachloro- and hexafluorocyclohexane isomers. Bull. Chem. Technol. Macedonia 23, 131-137 (2004).

14. Ogawa, S., Oki, S., Suami, T. Inositol Derivatives. 11. Synthesis of Dianhydroinositols. Bull. Chem. Soc. Jpn. 52, 1095- 1111 (1979). 
15. Lal., G. S., Pez, G. P., Pesaresi, R. J., Prozonic, F. M. Bis(2-methoxyethyl)aminosulfur trifluoride: a new broad-spectrum deoxofluorinating agent with enhanced thermal stability. Chem. Commum. 215-216 (1999).

16. Middleton, W. J. New fluorinating reagents. Dialkylaminosulfur fluorides. J. Org. Chem. 40, 574-578 (1975).

17. Beaulieu, F. et al. Aminodifluorosulfinium tetrafluoroborate salts as stable and crystalline deoxofluorinaing reagents. Org. Lett. 11, 5050-5053 (2009).

18. Schmidt, M. W. et al. General atomic and molecular electronic structure system. $J$. Comput. Chem. 14, 1347-1363 (1993).

19. Gordon, M. S., Schmidt, M. W. Advances in electronic structure theory: GAMESS a decade later. Theory and Applications of Computational Chemistry, the first forty years. Ch. 41 (Elsevier, Amsterdam, 2005).

20. Dunitz, J. D. Organic fluorine: odd man out. ChemBioChem 5, 614-621 (2004).

21. Kashtiban, R. J. et al. Atomically resolved imaging of highly ordered alternating fluorinated grapheme. Nature Commun. 5, 4902 (2014).

22. Sahin, H., Topsaka, M., Ciraci, S. Structures of fluorinated graphene and their signatures. Phys. Chem. Rev. B 83, 115432-115436 (2011).

23. Perrin, C. L., Dwyer, T. J. Application of two-dimensional NMR to kinetics of chemical exchange. Chem. Rev. 90, 935-967 (1990).

24. Anet, F. A. L., Bourn, A. J. R. Nuclear magnetic resonance line-shape and doubleresonance studies of ring inversion on cyclohexane-d ${ }_{11}$. J. Am. Chem. Soc. 89, 760-798 (1967).

25. Badenhoop, J. K., Weinhold, F. Natural bond order analysis of steric interactions, $J$. Chem. Phys. 107, 5406-5421 (1997). 
26. Glendening, E. D., Landis, C. R., Weinhold, F. NBO 6.0: Natural Bond Orbital analysis program. J. Comput. Chem. 34, 1429-1437 (2013).

27. Reed, A. E., Curtiss, L. A., Weinhold, F. Intermolecular interactions from a Natural Bond Orbital, donor-acceptor viewpoint. Chem. Rev. 88, 899-926 (1988).

28. Weinhold, F., Landis, C. R. Valency and Bonding: A Natural Bond Orbital DonorAcceptor Perspective (Cambridge University Press, U.K., 2005).

29. Nelson, R. D., Lide, D. R., Maryott, A. A. Selected values of electric dipole moments for molecules in the gas phase. Natl. Stand. Ref. Data Ser. - Nat. Bur. Stnds. 10 (1967).

30. Haynes, W. M. CRC Handbook of Chemistry and Physics 95th Ed. Section 9-15 (CRC Press, Boca Raton, 2014).

31. Kawai, H. The piezoelectricity of poly (vinylidine fluoride). Japan J. Appl. Phys. 8, 975$976(1969)$.

32. Goodman, L., Gu, H., Pophristic, V. Gauche effect in 1,2-difluoroethane. Hyperconjugation, bent bonds, steric repulsion. J. Phys. Chem. A 109, 1223-1229 (2005).

33. Patrick, C. R., Prosser, G. S. A molecular complex of benzene and hexafluorobenzene. Nature 187, 1021 (1960).

34. Williams, J. H., Cockroft, J. K., Fitch, A. N. Structure of the lowest temperature phase of the solid benzene-hexafluorobenzene adduct. Angew. Chem. Int. Ed. 31, 1655-1657 (1992). 


\section{Acknowledgments}

This work was generously supported by the Engineering and Physical Sciences Research Council (EPSRC) and the European Research Council (ERC). D.O'H. thanks the Royal Society for a Wolfson Research Merit Award. The authors acknowledge the EPSRC National Mass Spectrometry Facility (Swansea) for analytical assistance.

\section{Author contributions}

N.K. and D.O'H conceived the synthesis of 1. N.K. designed the synthetic route and performed all of the synthesis and standard characterization. A.M.Z.S. obtained and solved the crystallographic data. T.L. conducted the low temperature EXSY NMR studies and analyzed the data. D.P. carried out the DFT and NBO theory studies. The manuscript was cowritten by N.K. and D.O'H. All authors discussed the results and commented on the manuscript.

\section{Additional information}

Supplementary information and chemical compound information are available in the online version of the paper. Reprints and permissions information is available online at www.nature.com/reprints. Correspondence and requests for materials should be addressed to D. O’H.

\section{Competing financial interests}

The authors declare no competing financial interests. 


\section{Figures}

a

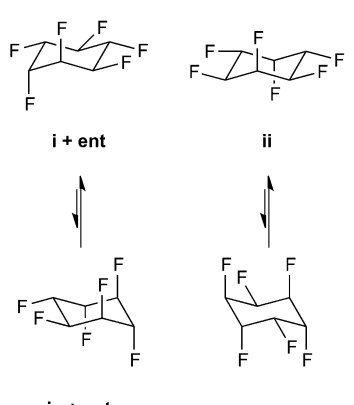

ix + ent

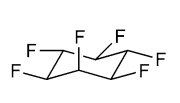

iii

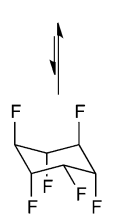

$\mathbf{x i}$

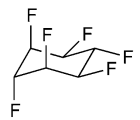

iv

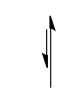

degenerate

ing

same
conformation conformationa
enantiomer
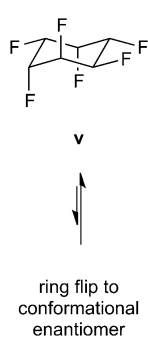

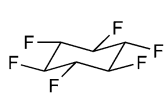

vi

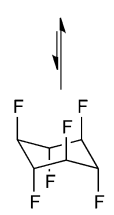

xii
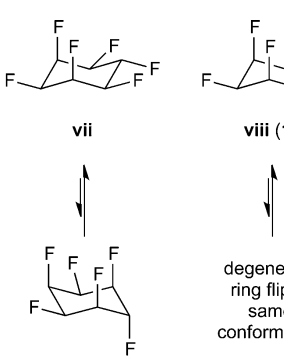

degenerate ring flip to same

xiii
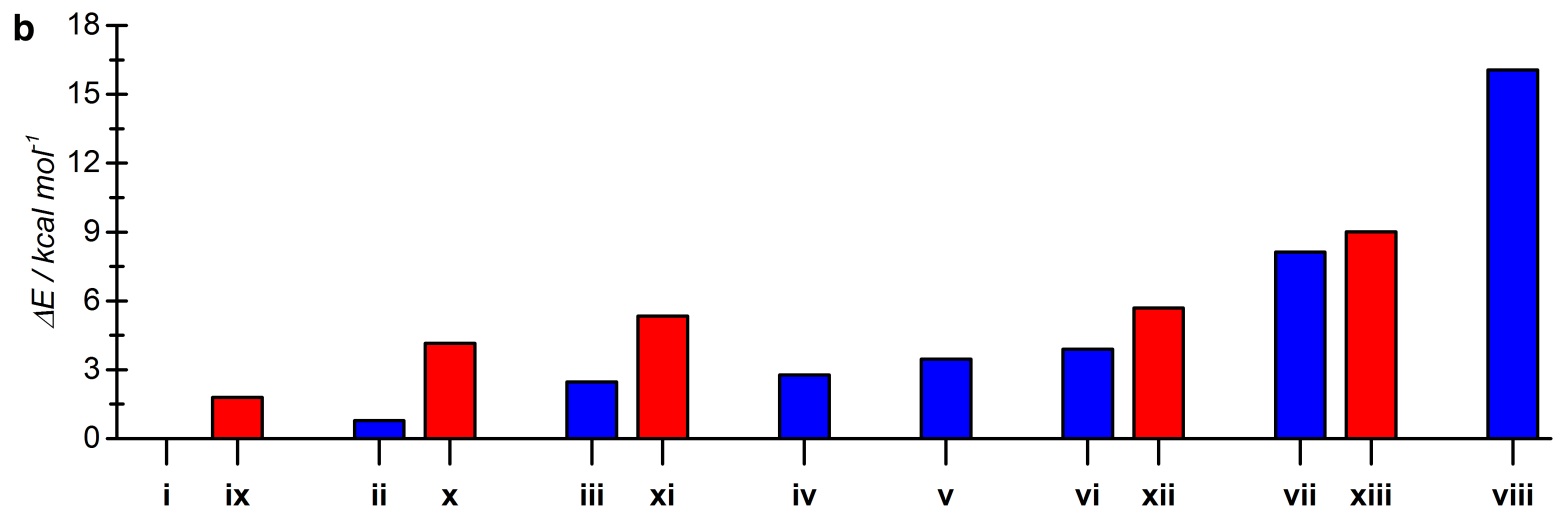

Figure 1 | The structures and relative energies of all isomers of 1,2,3,4,5,6-hexafluorocyclohexane. a, The chair structures of all the different isomers ( 9 configurational and a total of 15 possible conformational isomers) of 1,2,3,4,5,6-hexafluorocyclohexane are illustrated. b. Histogram format of the calculated (MP2/6$31++G)^{13}$ relative ground state energies of each isomer in $\mathrm{kcal} \mathrm{mol}^{-1}$. They are shown in ascending order of their ground state chair structures; the lowest energy isomer being $\mathbf{i}\left(0.00 \mathrm{kcal} \mathrm{mol}^{-1}\right)$. Blue bars denote the lowest energy configurational isomers and red bars indicate the higher energy conformational isomers, when a particular configurational isomer undergoes a ring flip between alternative chair structures. Structures iv, $\mathbf{v}$ and viii have a single blue bar which denotes that their corresponding ring flip structures are degenerate (iv, $\mathbf{v}$ and $\mathbf{v i i i}$. The ring flip of $\mathbf{v}$ is degenerate, but produces the enatiomeric structure. 
<smiles>OC1C(O)C2C(O)C(O)C(C1O)C2O</smiles>

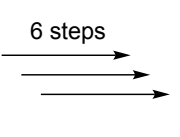<smiles>O[C@@H]1[C@@H]2O[C@@H]2[C@@H]2O[C@@H]2[C@@H]1O</smiles>

3

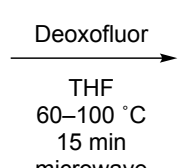

microwave

94\%<smiles>FC1C(F)[C@H]2O[C@H]2[C@@H]2O[C@H]12</smiles>

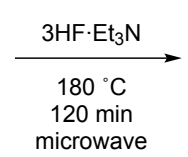

$71 \%$<smiles>O[C@H]1C(F)[C@H](F)[C@H](F)[C@H](F)[C@H]1F</smiles>

5<smiles>FC1C(F)C(F)C(F)C(F)C1F</smiles>

1

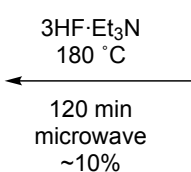

$\sim 10 \%$<smiles>[O]C1C(F)C(F)C(F)C(F)C1F</smiles>

8

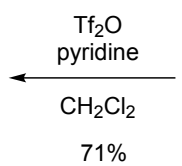

$71 \%$<smiles>O[C@H]1C(F)[C@H](F)C(F)[C@H](F)C1F</smiles>

7

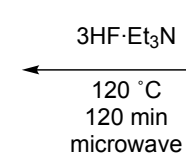

$40 \%$<smiles>O[C@H]1C(F)[C@@H](O)C(F)[C@H](F)C1F</smiles>

6

Figure 2 | Synthetic route to all-cis 1,2,3,4,5,6-hexafluorocyclohexane 1. The route commenced from myoinositol 2, which was converted to diepoxidediol $\mathbf{3}$ over 6 steps (see Supplementary Information) following known chemical transformations. This afforded $\mathbf{3}$ as the source of an appropriately functionalised cyclohexane with all of the $\mathrm{C}-\mathrm{O}$ bonds on one face of the cyclohexane ring. The synthesis of $\mathbf{1}$ was then achieved by progressing through a sequence of deoxyfluorination reactions occurring with an inversion of configuration at each carbon in order to achieve the desired all-cis arrangement of the fluorine atoms around the ring. 


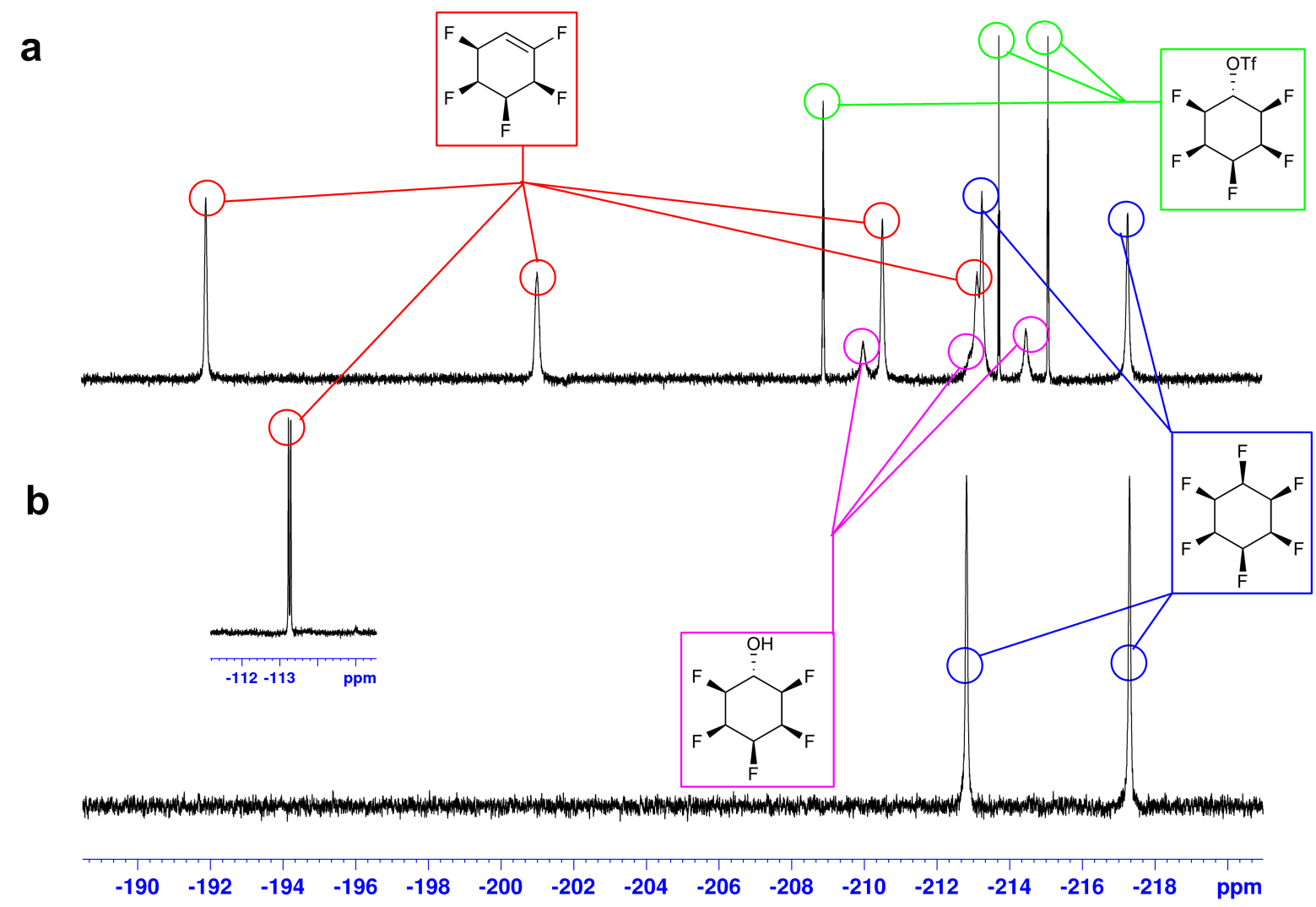

Figure $3 \mid{ }^{19} \mathbf{F}\left\{{ }^{1} \mathbf{H}\right\}$ NMR spectra of all-cis $\mathbf{1 , 2}, \mathbf{3}, \mathbf{4 , 5 , 6}$-hexafluorocyclohexane 1. a, A partial ${ }^{19} \mathrm{~F}\left\{{ }^{1} \mathrm{H}\right\} \mathrm{NMR}$ spectra ( $470.6 \mathrm{MHz}, \mathrm{CD}_{2} \mathrm{Cl}_{2}, 298 \mathrm{~K}$ ) (with inset) of the product mixture arising from the last synthesis step in the conversion of $\mathbf{8}$ to $\mathbf{1}$. Three co-produced products $(\mathbf{1}, \mathbf{7}$ and pentafluoro alkene) are formed (in addition to residual starting material 8) and the signals are assigned to the structures as shown. $\mathbf{b}$, The ${ }^{19} \mathrm{~F}\left\{{ }^{1} \mathrm{H}\right\} \mathrm{NMR}$ spectra ( $470.6 \mathrm{MHz}, \mathrm{CD}_{2} \mathrm{Cl}_{2}, 298 \mathrm{~K}$ ) of purified $\mathbf{1}$ after chromatography showing the non equivalent axial and equatorial fluorine environments. 

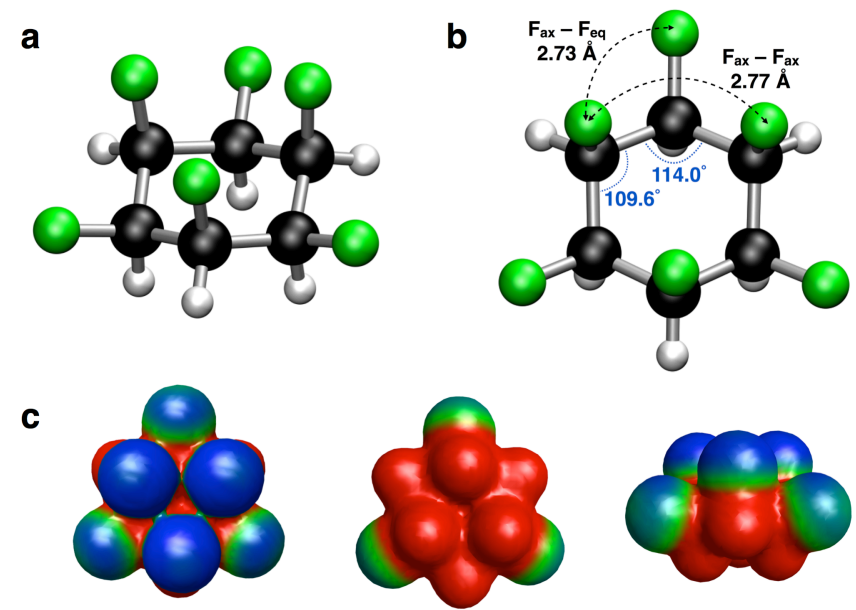

Figure 4 | Different representations of all-cis 1,2,3,4,5,6-hexafluorocyclohexane 1. a and $\mathbf{b}$ show two orientations of the single crystal $\mathrm{X}$-ray structure of 1 illustrating $\mathbf{a}$, the chair conformation with alternating axial and equatorial $\mathrm{C}-\mathrm{F}$ bonds and $\mathbf{b}$, a top down view of the structure highlighting the mean distances between fluorine atoms and the alternating variation of the mean $\mathrm{C}-\mathrm{C}-\mathrm{C}$ angles around the ring. All distance and angle measurements from the single crystal structure are shown in Supplementary Figure 2 (page 28). Notably the ring is not distorted and the three triaxial $\mathrm{C}-\mathrm{F}$ bonds are close to parallel. c, Electrostatic potential surface maps derived from an M11/6-311G(2d,p) electronic structure calculation, show that the molecule is highly polarised with a -ve (blue) and a +ve (red) face. The fluorine (blue -ve) and hydrocarbon (red $+v e)$ faces are colour contrasted in top bottom and side view profiles of $\mathbf{1}$. 


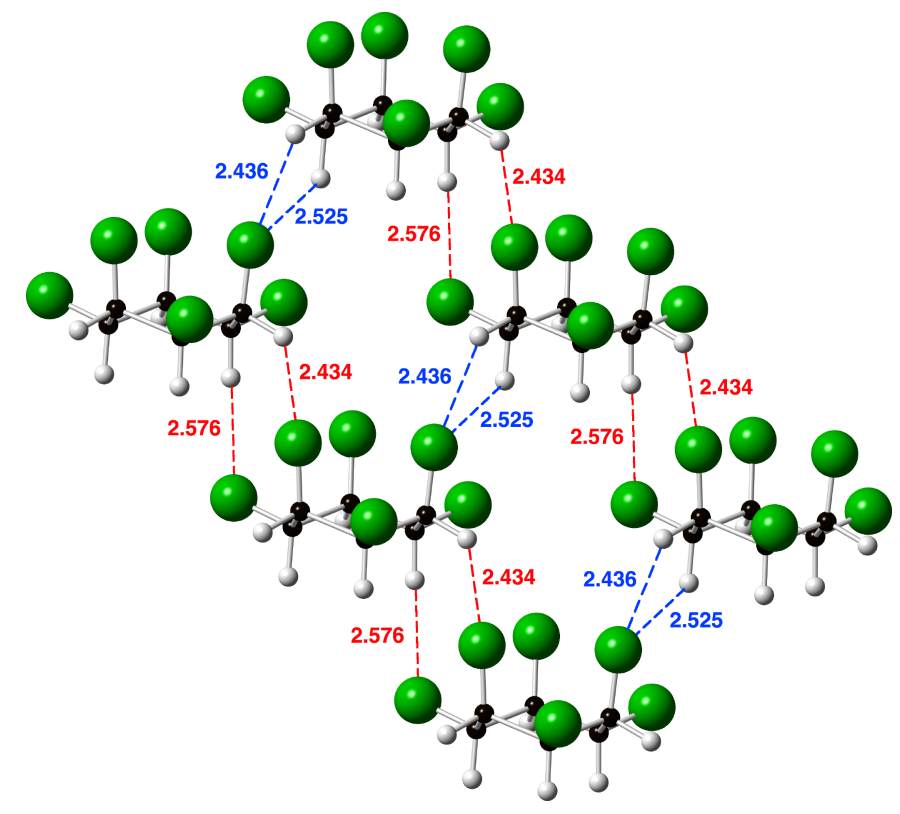

Figure 5 | Representation of the crystal packing structure of 1, derived from the single crystal X-Ray data. The shortest intermolecular distances between molecules are shown in Angstroms $(\AA)$, highlighting the key intermolecular interactions. The molecules arrange consistent with facial polarity and electrostatic ordering with negatively-charged fluorine faces contacting the positively-charged hydrogen faces of adjacent molecules, rather than an alternative mode of packing where fluorous-fluorous and hydrocarbonhydrocarbon faces could aggregate together. 


\begin{tabular}{|c|c|c|}
\hline Donor $(\mathbf{L}) \mathbf{N B O}$ & $\begin{array}{c}\text { Acceptor (NL) } \\
\text { NBO }\end{array}$ & Energy $/ \mathbf{k c a l ~ m o l} \mathbf{~}^{\mathbf{1}}$ \\
\hline$n\left(\mathrm{~F}_{\text {ax }}^{1} \mathrm{LP}^{1}\right)$ & $\mathrm{C}^{1}$ Rydberg & 7.5 \\
\hline$n\left(\mathrm{~F}_{\text {ax }}^{1} \mathrm{LP}^{2}\right)$ & $\sigma^{*} \mathrm{C}^{1}-\mathrm{H}^{1}$ & 10.1 \\
\hline$n\left(\mathrm{~F}_{\text {ax }}^{1} \mathrm{LP}^{3}\right)$ & $\sigma^{*} \mathrm{C}^{1}-\mathrm{C}^{2}$ & 7.8 \\
\hline$n\left(\mathrm{~F}_{\text {ax }}^{1} \mathrm{LP}^{3}\right)$ & $\sigma^{*} \mathrm{C}^{1}-\mathrm{C}^{6}$ & 7.7 \\
\hline$n\left(\mathrm{~F}_{\text {eq }}^{2} \mathrm{LP}^{1}\right)$ & $\mathrm{C}^{2} \mathrm{Rydberg}$ & 7.5 \\
\hline$n\left(\mathrm{~F}_{\text {eq }}^{2} \mathrm{LP}^{2}\right)$ & $\sigma^{*} \mathrm{C}^{2}-\mathrm{H}^{2}$ & 10.6 \\
\hline$n\left(\mathrm{~F}^{2}{ }_{\text {eq }} \mathrm{LP}^{3}\right)$ & $\sigma^{*} \mathrm{C}^{2}-\mathrm{C}^{1}$ & 7.0 \\
\hline$n\left(\mathrm{~F}^{2}{ }_{\text {eq }} \mathrm{LP}^{3}\right)$ & $\sigma^{*} \mathrm{C}^{2}-\mathrm{C}^{3}$ & 6.9 \\
\hline$\sigma \mathrm{C}^{2}-\mathrm{H}^{2}$ & $\sigma^{*} \mathrm{C}^{1}-\mathrm{F}^{1}$ & 6.3 \\
\hline$\sigma \mathrm{C}^{2}-\mathrm{H}^{2}$ & $\sigma^{*} \mathrm{C}^{3}-\mathrm{F}^{3}$ & 6.3 \\
\hline
\end{tabular}

Table 1 | Natural Bond Orbital (NBO) interaction energies (NBO 6.0, M11/6-311G(2d,p) theory level) calculated for the axial and equatorial fluorine atoms of 1. The data gives an insight into the electron distribution of the three fluorine lone pairs (two with p-orbital character and one with s-orbital character) within the molecule, and particularly how the electron distribution adapts to placing the ax/eq and ax/ax fluorine atoms within close proximity to each other. The most stabilising interactions for both the axial and equatorial fluorines involve dispersion of the electron density of one lone pair ( $L P^{3}, p$-character) into two adjacent $\mathrm{C}-\mathrm{C}$ antibonding orbitals. The $\mathrm{F}$ lone pairs with s-character, interact with the diffuse Rydberg state on the attached carbon atom. The full table of NBO interactions, and diagrammatic representations of the NBOs in Table 1 are shown in the Supplementary Table 1 and Figure 5 (page 36-37). 


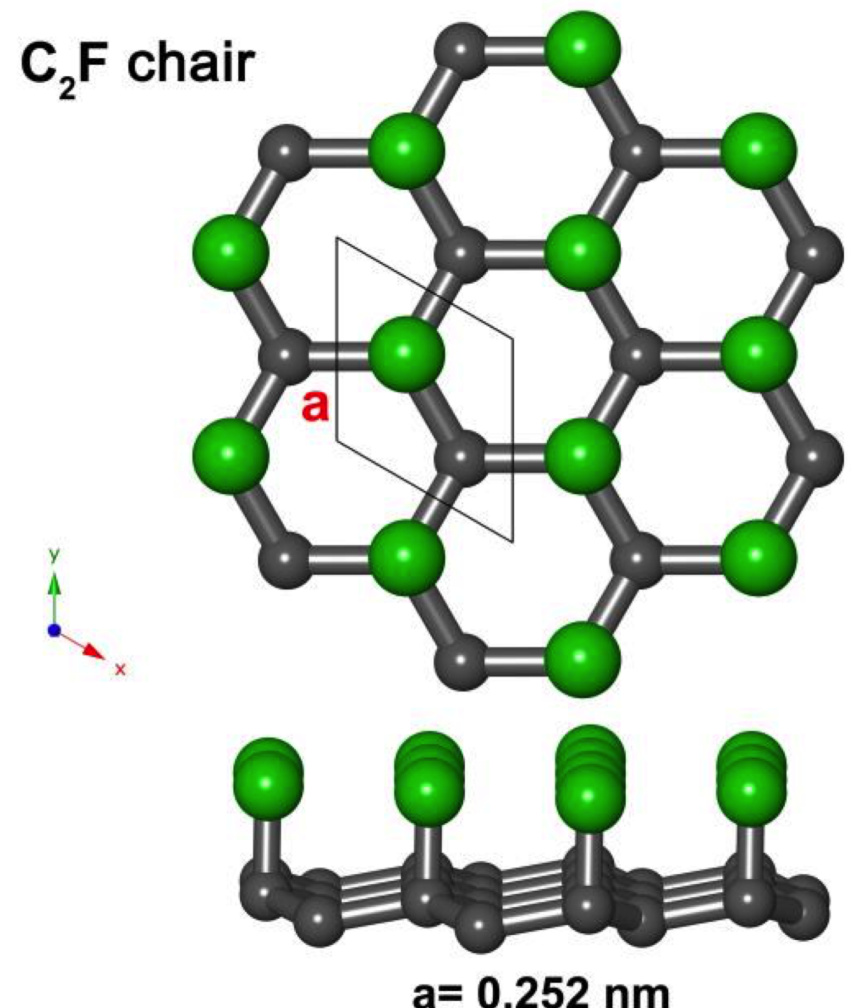

Figure 6 | Representation of the 2D structure of $\mathbf{C}_{\mathbf{2}} \mathbf{F}$ chair graphene. ${ }^{21}$ This structure was determined by transmission electron microscopy after the partial fluorination of graphene with $\mathrm{XeF}_{2}$. Exit wave reconstruction (EWR) images suggest fused six membered rings in a chair conformation with the fluorines attached to alternate carbons and only on one face of the graphene. There are no hydrogens in the structure. The idealised structure has rings with three axial C-F bonds and therefore has some analogy with the structure of $\mathbf{1}$. Image by permission from Nature Commun. ${ }^{21}$. 